\title{
Composition Dependence of Fluid Thermophysical Properties: Theory and Modeling
}

DoE Grant DE FG02-92ER14294

Submitted by

James F. Ely

Chemical Engineering and Petroleum

Refining Department

Colorado School of Mines

Golden, CO 80401

Office: (303) 273-3885

Telefax: (303) 273-3730

EMAIL: JELY@MINES.COLORADO.EDU

March 29, 1993

Administrative Contact: Mary Mittag-Miller

(303) 273-3411

\section{DISCLAIMER}

\footnotetext{
This report was prepared as an account of work sponsored by an agency of the United States Government. Neither the United States Government nor any agency thereof, nor any of their employees, makes any warranty, express or implied, or assumes any legal liability or responsibility for the accuracy, completeness, or usefulness of any information, apparatus, product, or process disclosed, or represents that its use would not infringe privately owned rights. Reference herein to any specific commercial product, process, or service by trade name, trademark, manufacturer, or otherwise does not necessarily constitute or imply its endorsement, recommendation, or favoring by the United States Government or any agency thereof. The views and opinions of authors expressed herein do not necessarily state or reflect those of the United States Government or any agency thereof.
} 


\section{Contents}

1 Introduction $\quad 1$

2 Summary of Research Findings $\quad 1$

3 Progress 2

3.1 Computer Simulation Studies . . . . . . . . . . . . . . . . . 2

3.2 Selection Algorithm Methodology . . . . . . . . . . . . . . . . 3

3.3 Advanced Theories of Fluid Mixtures . . . . . . . . . . . . . . 6

4 Summary $\quad 9$

5 Publications and Presentations $r$

5.1 Published Manuscripts $8 / 1 / 92-7 / 31 / 93 \ldots \ldots \ldots \ldots$

5.2 Manuscripts Submitted or In Press . . . . . . . . . . . . . 10

5.3 Manuscripts in preparation . . . . . . . . . . . . . . . . 10

5.4 Presentations, $8 / 1 / 92-7 / 31 / 93 \ldots \ldots \ldots \ldots \ldots$

6 Budget $\quad 12$

7 Estimated Unexpended Balance $\quad 13$

\section{List of Figures}

1 Simulation results for the Kirkwood charging integrals . . . . . . . . . . . 4

2 Simulation results for the excess Gibbs free energy of a Lennard- Jones mixture 5 


\section{Introduction}

In this report, we highlight the progress made and expected during the period August 1, 1992 through July 31, 1993 on DoE Grant DE-FG02-92ER14294. Since the latter date is several months away, we include some discussion of work in progress and expected results, although we primarily concentrate on work that has been completed. The objectives of this research are four-fold:

1) Fundamental investigation of the equilibrium and nonequilibrium properties of asymmetric fluid mixtures through computer simulation;

2) Development of advanced, highly accurate predictive theories of mixture equilibrium properties;

3) Development and application of selection algorithm methodology and results from fundamental theory to the design of high accuracy mixture equations of state;

4) Application of new theoretical concepts and insights to the development of engineering design models.

Significant progress is being been made in all of these areas as is described in the next two sections. In addition, publications presenting the results of our ongoing DoE sponsored studies are in press or have appeared during the reporting period. These are summarized in the last section.

\section{Summary of Research Findings}

1) Kirkwood charging method computer simulations of systems of Lennard-Jones mixtures with large size ratios have clarified the the low composition dependence of the coupling parameter and have conclusively verified the accuracy of our Kirkwood-Buff/Baxter method of calculating chemical potentials.

2) Computer simulations of $n$-butane have shown that the rheological behavior reported earlier is not a function of system size.

3) We have developed a modified step-wise regression algorithm for determining the functional dependence of equations of state for complex systems. The method has been successfully applied to the development of the most accurate (to date) equation of state for HFC R.134a.

4) We have developed an analytic expression for the conformal solution size correction for mixtures. The analytic model is being used to develop a true mixture equation of state.

5) We have discovered that the extended corresponding states theory can be successfully applied to systems which have large polarity differences. In particular, we have developed an accurate representation of the bulk phase properties of water-hydrocarbon systems. 
6) We discovered how to force the extended corresponding states theory (ECST) to reach the correct second virial limit. This enables one to make more accurate predictions of phase equilibria with the ECST approach.

\section{Progress}

In this section we report in narrative form progress that has been attained during the contract period of performance. There are three general areas discussed: 1) Computer Simulation, 3) Selection algorithm methodology, and 3) Advanced corresponding states theories for mixture properties. Our work on the development of engineering design models is included in the discussion on item 3 ).

\subsection{Computer Simulation Studies}

A key component of our research program is to use computer simulation to systematically investigate the effects of size, shape, polarity and external disturbances (e.g., shear) on thermophysical properties. During the reporting period, we have continued our computer simulation studies of the equilibrium properties of systems with large size differences. In addition we have performed some non-equilibrium simulations on structured molecules. The primary equilibrium problem that has been studied is that of the difficulty of simulating entropic properties in mixtures where there are large size ratios. In particular, Monte Carlo based methods (particle insertion methods) fail in these systems due small acceptance ratios when the size difference is large.

To overcome this problem we have developed a method where we use the direct correlation function integral form (DCFI) of the Kirkwood-Buff (KB) theory to obtain entropic properties and molecular dynamics simulation results for the radial distribution functions to obtain the required DCFI's. This approach is particularly novel since it is the first to incorporate Baxter's factorization of Ornstein-Zernicke (OZ) equation to obtain DCFI's from simulation data. Our initial comparisons of the new method with results from the Percus-Yevick theory and Haile's adaptation of the Kirkwood charging method were encouraging although there were significant differences in the low concentration region of the small molecule.

During the reporting period we have performed extensive simulations using the Kirkwood charging method to elucidate the apparent differences which were observed in our initial comparisons. In the Kirkwood charging method the excess Gibb's free energy is calculated from

$$
g^{E}=x_{A} \int_{1}^{\lambda_{m}}<F_{A A}>d \lambda+x_{B} \int_{1}^{\lambda_{m}}<F_{B B}>d \lambda
$$

where

$$
\begin{gathered}
F_{A A}=\frac{f_{A B}}{1+\lambda}+\frac{f_{B B}}{\lambda} \\
F_{B B}=-\frac{f_{A B}}{\lambda(1+\lambda)}-\frac{f_{A A}}{\lambda}
\end{gathered}
$$


and

$$
f_{\alpha \beta}=24\left(2-\delta_{\alpha \beta}\right) \frac{1}{N} \sum \sum \epsilon_{\alpha \beta}\left[2\left(\sigma_{\alpha \beta} / \gamma_{i j}\right)^{12}-\left(\sigma_{\alpha \beta} / r_{i j}\right)^{6}\right]
$$

The charging parameter is defined as $\lambda=\sigma_{B B} / \sigma_{A A}$. In order to calculate the excess Gibbs free energy using this method, a total of 11 simulations were performed for each of 7 compositions, e.g., a total of 77 simulations. The temperature in these simulations was taken to be $240 \mathrm{~K}$ and the pressure was 209 bar. Equal energies were assumed (e.g., $\epsilon_{A A}=\epsilon_{B B}$ ) and the nominal molecular size ratio was two. Each simulation was carried out for 40,000 MD time-steps using an IBM RS/6000, Model 320H computer (acquired with DoE support). Figure 1 shows the simulation results for the quantities $\left\langle F_{A A}\right\rangle$ and $\left\langle F_{B B}\right\rangle$ and Figure 2 shows the results of the excess Gibb's free energy calculations for the three methods investigated: Percus-Yevick KB Method, $\mathrm{MD} / \mathrm{KB}$ method and the Kirkwood charging method. The agreement between the simulation methods is excellent, indicating that the MD/KB method is an accurate method for obtaining chemical potentials in systems with large size ratios. The advantage of the $\mathrm{MD} / \mathrm{KB}$ method is that only 7 (as opposed to 77) simulations are required to generate the excess Gibbs free energy curve.

\subsection{Selection Algorithm Methodology}

A new component of our research is the improvement and application of selection algorithm methodology for the development of mixture equations of state. Our overall objectives in this area are:

1) Design and develop an optimal corresponding states reference fluid equation of state which only incorporates temperature dependence up to $\mathrm{T}^{3}$, i.e., the temperature dependent terms come from the set $\left\{T^{ \pm \frac{n}{2}}\right\}$ where $|n| \leq 6$. In effect, this effort is to find the best reference fluid equation of state based on the known characteristics of the mixing rules.

2) Develop super high accuracy equations of state for selected pure fluids by superimposing rescaling or crossover theory on the selection algorithm. The net result of this study will be more compact, higher accuracy equations of state for fluids where sufficient data exist to warrant definitive correlation.

3) Directly use the selection algorithm to develop a mixture equation of state with explicit composition dependence. It is envisioned that the resulting equation could be used both as the (correlative) background function in a crossover model and as a mixture reference fluid in an advanced corresponding states model.

The first step in this study is to develop the selection algorithm methodology. During the reporting period two selection algorithm programs have been developed. The first uses the simulated annealing method in which a cost function obtained from multiproperty linear regression is minimized. The method was successfully applied to the determination of a new function for the vapor pressure. Five fluids were simultaneously correlated from a bank of 100 terms. The optimal representation used 7 of the 100 terms and included a constraint at the critica: point. Surprisingly, the scaling contribution to the vapor pressure, $\tau^{2-\theta}$ was not selected. 

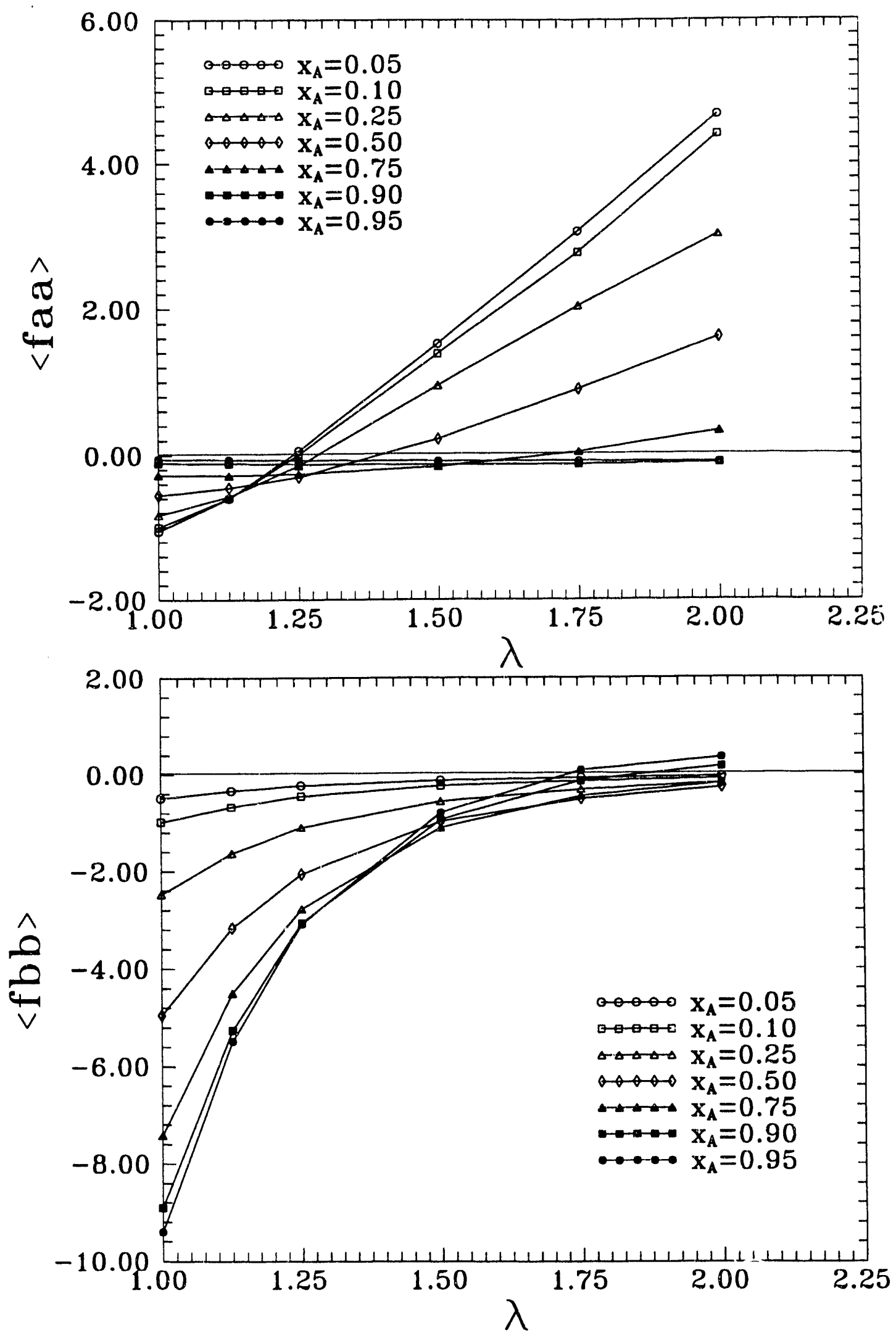

Figure 1: Simulation results for the $\left\langle F_{A A}\right\rangle$ and $\left\langle F_{B B}\right\rangle$ Kirkwood charging integrands for seven compositions of a 2:1 Lennard-Jones fluid 


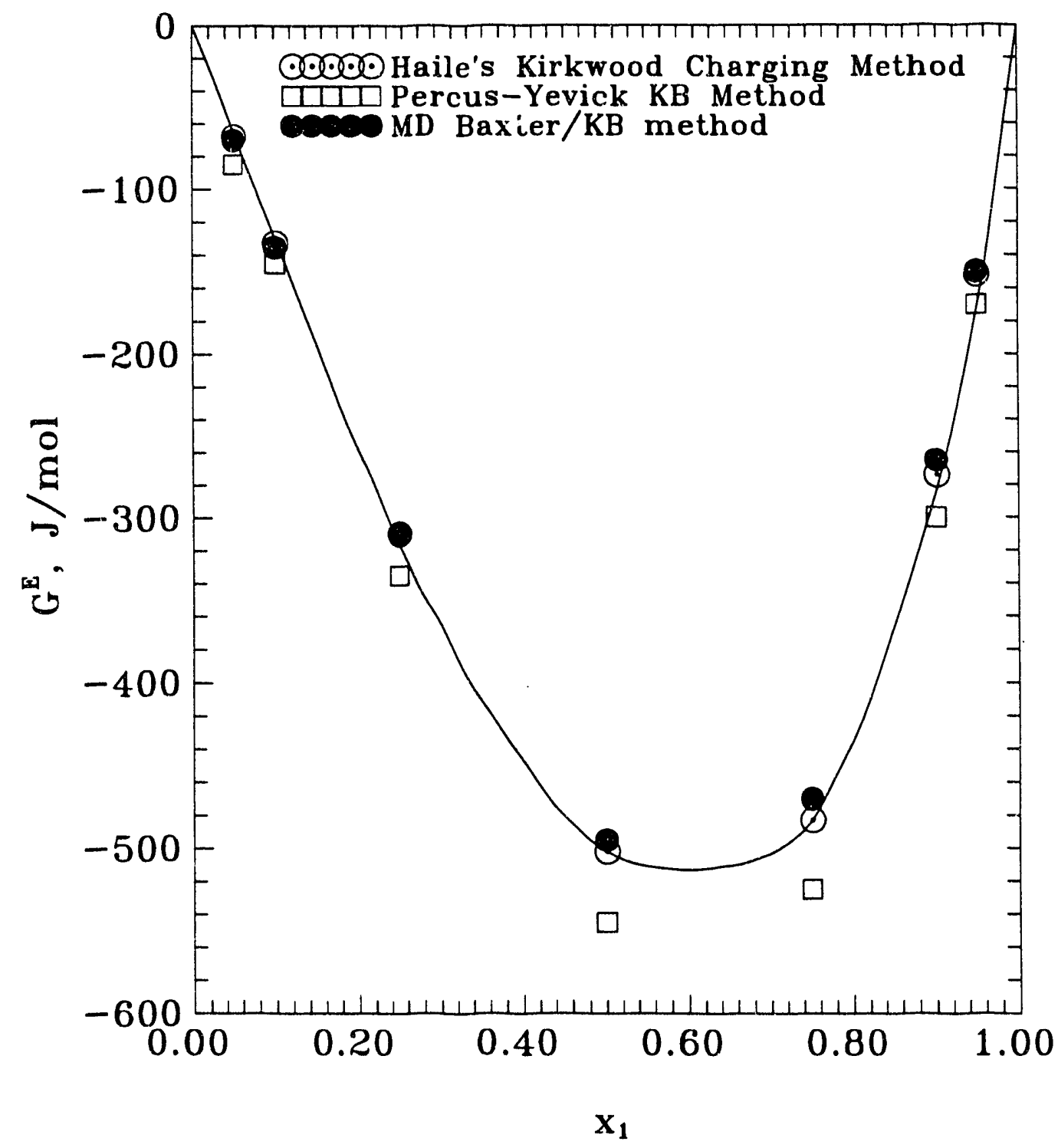

Figure 2: Simulation results for the excess Gibbs free energy of Lennard-Jones mixtures as a function of composition. $\left\langle\sigma_{A A} / \sigma_{B B}\right\rangle=2$ and $\left.\epsilon_{A A} / \epsilon_{B B}\right\rangle=1$. 
The second algorithm which was developed utilizes a step-wise regression procedure with a superimposed stochastic mutation scheme. This procedure which is faster than the simulated annealing algorithm and has been used to develop a new equation of state for the HFC refrigerant R134a. R134a was selected for this study because of its commercial importance and energy conservation/utilization impact. The new equation is applicable to both liquid and vapor phases at pressures up to $70 \mathrm{MPa}$ with temperatures from the triple point to $450 \mathrm{~K}$. The functional form of the equation is in terms of the Helmholtz free energy as a function of reduced temperature and density,

$$
\frac{A^{r}}{R T}=\sum_{\alpha=1}^{28} c_{\alpha} \delta^{i_{\alpha}} \tau^{j_{\alpha}} e^{-\delta^{k_{\alpha}}}
$$

where $\delta=\rho / \rho_{c}, \tau=T_{c} / T$ and the superscript $r$ indicates a residual relative to the ideal gas at the same temperature and density. The $c_{\alpha}$ are determined from the multiproperty, stepwise regression process using experimental PVT, heat capacity, second virial, isothermal compressibility and saturation data. To the best of our knowledge, this equation is the most accurate equation of state available for this fluid.

Work is underway to restrict the temperature dependence of the equation as described in 1) and to incorporate the rescaling algorithm as described in 2). In particular, we expect to have a rescaled equation of state by the end of the reporting period.

\subsection{Advanced Theories of Fluid Mixtures}

Conformal solution or corresponding states theories have been selected as the core of our predictive modeling research since this type of model has proven to be (overall) the most accurate and the most widely applicable to different types of fluids. The general objectives of this portion of our proposed research are:

1) Improve and extend molecular based corresponding states models to more complex systems.

2) Extend the VDW-HSC model for size difference effects to simpler equations of state (e.g., Carnahan-Starling-deSantis, Peng-Robinson, etc).

3) Develop a corresponding states principle based on integral equation formalism thereby capturing the implicit composition dependence contained in the Ornstein-Zernicke equation.

Excellent progress has already been made on items 1) and 2) and work is underway on item 3 ). In the area of improving corresponding states models [item (1)] we have been able to solve one long standing problem, namely how one-fluid models approach the second virial limit. In addition we have successfully applied the modified extended corresponding states model to highly asymmetric water/hydrocarbon and water/carbon dioxide systems.

As summarized in our original proposal, the behavior of one-fluid theories in the second virial limit has serious impact on the accuracy of vapor-liquid equilibria. In particular, we found that: 
1) Errors in the extended corresponding states VDW-1 model K-values come from improper mapping of the mixture second virial coefficient onto the reference fluid. The reason this occurs is that the VDW-1 mixing rules are accurate only to the power of $1 / \mathrm{T}$.

2) In the near critical region of the mixture, the calculated vapor phase fugacity coefficients (and VLE predictions) are dominated by how the reference fluid equation of state extrapolates to high temperatures where the second virial contribution is dominant. This problem is particularly acute as the mixture components become more disparate.

We have discovered a simple but effective way of minimizing these problems by extending the ideas of Sandler and Wong to the more complex VDW-1 extended corresponding states theory using exact shape factors. In the unmodified VDW-1 theory one uses the mixing rules

$$
h_{x}=\sum_{i=1}^{n} \sum_{j=1}^{n} x_{i} x_{j} h_{i j}
$$

and

$$
f_{x} h_{x}=\sum_{i=1}^{n} \sum_{j=1}^{n} x_{i} x_{j} f_{i j} h_{i j} .
$$

where $f_{x}$ and $h_{x}$ are conformal solution energy and volume scale factors. In the second virial limit one finds that $B_{m i x}$ is given by

$$
B_{m i x}=h_{x} B_{0}\left(T / f_{x}\right)=\sum_{i} \sum_{j} x_{i} x_{j} h_{i j} B_{0}\left(T / f_{x}\right)
$$

where the subscript 0 denotes the reference fluid. This result is exact if the second virial coefficient on depends upon the zero-th or first power of $T$. Otherwise errors are introduced which are amplified as the mixture components become more asymmetric. Our proposed modification is to replace equation (6) with a form which is exact in the second virial limit, viz.

$$
h_{x}=\frac{\sum_{i} \sum_{j} x_{i} x_{j} h_{i j} B_{0}\left(T / f_{i j}\right)}{B_{0}\left(T / f_{x}\right)}
$$

Obviously, if the unmodified VDW-1 model was exact in this limit, we would have $B_{0}\left(T / f_{x}\right)=B_{0}\left(T / f_{i j}\right)$ and equation (9) would be identical to equation (6). We are currently evaluating the predictions obtained with this new mixing rule in near-critical aqueous mixtures containing carbon dioxide. The preliminary results are encouraging and we expect to present a paper summarizing this work at the 13th International European Conference on Thermophysical Properties in Lisbon in August and the National AIChE meeting in St. Louis in November.

Another result obtained from our efforts to improve corresponding states theories is an analytical approximation to the size correction term in the VDW-HSC model developed previously by Huber and Ely. The essential idea in this improved corresponding states theory is the use perturbation theory to correct conformal solution theory rather than to try to correct the rather unphysical properties of some hard convex body which is used as 
the reference material in conventional perturbation theory. The derivation of the theory has been published with the final result being

$$
\frac{A_{m i x}^{r}}{R T}=\frac{A_{x}^{r}}{R T}+\frac{2 \pi \rho}{k T}\left[I_{m i x}-I_{x}\right]
$$

where

$$
I_{m i x}=\sum_{i} \sum_{j} x_{i} x_{j} \int_{d_{i j}}^{\infty} u_{i j}(r) g_{i j}^{H S}\left(r,\left\{\rho_{k}\right\},\left\{d_{k l}\right\}\right) r^{2} d r
$$

and

$$
I_{x}=\int_{d_{x}}^{\infty} u_{x}(r) g_{x}^{H S}\left(r, \rho, d_{x}\right) r^{2} d r
$$

In order to apply this model, the effective hard sphere diameters $d_{x}$ and $\left\{d_{i j}\right\}$ must be specified. We have set the $\left\{d_{i j}\right\}$ equal to the Lennard-Jones $\left\{\sigma_{i j}\right\}$, and calculated $d_{x}$ from

$$
d_{x}^{3} g_{x}^{H S}\left(d_{x}\right) \sum_{i=1}^{n} \sum_{j=1}^{n} x_{i} x_{j} d_{i j}^{3} g_{i j}^{H S}\left(d_{i j}\right)
$$

In the original implementation of this model, the integrals appearing in equations (11) and (12) were evaluated numerically. This procedure, although straightforward, makes the model computationally intractable for routine use. We have, therefore, developed an analytical approximation to these integrals.

The approximation is obtained by re-expressing the integrals in terms of the simpler Laplace transforms of the distribution function and potential, viz.

$$
I_{m i x}=\sum_{i} \sum_{j} x_{i} x_{j} \int_{0}^{\infty} U_{i j}(s) G_{i j}^{H S}(s) d s
$$

and

$$
I_{x}=\int_{0}^{\infty} U_{x}(s) G_{x}^{H S}(s) d s
$$

where the $G_{i j}^{H S}(s)$ are the Laplace transforms of $r g_{i j}^{H S}(r)$ and $U_{i j}(s)$ is defined by

$$
r u_{i j}(r)=\int_{0}^{\infty} U_{i j}(s) e^{-s r} d s
$$

In order to evaluate equations (14) and (15) we have made an approximation about the transform of the radial distribution functions. In particular, the transforms are given by

$$
G_{i j}^{H S}(s)=\frac{e^{-s \sigma_{i j}}}{s^{2} D \Delta}\left[s\left(\sigma_{i j}+\sigma_{i} \sigma_{j} \frac{\pi}{4 \Delta} \xi_{2}\right)+1+\frac{\pi}{2 \Delta} \xi_{3}+\frac{\pi}{2 \Delta} s \sum \rho_{k}\left(\sigma_{k}-\sigma_{i}\right)\left(\sigma_{k}-\sigma_{j}\right) \phi_{1}\left(\sigma_{k}\right)\right]
$$

where

$$
\begin{array}{r}
D(s)=1-\frac{2 \pi}{\Delta}\left(1+\frac{\pi}{2 \Delta} \xi_{3}\right) \sum \rho_{k} \phi_{2}\left(\sigma_{k}\right)-\frac{2 \pi}{\Delta} \sum \rho_{k} \sigma_{k} \phi_{1}\left(\sigma_{k}\right)\left(1+\frac{\pi}{4 \Delta} \xi_{2} \sigma_{k}\right) \\
-\frac{1}{2}\left(\frac{\pi}{\Delta}\right)^{2} \sum \sum \rho_{k} \rho_{l} \phi_{1}\left(\sigma_{k}\right) \phi_{1}\left(\sigma_{l}\right)\left(\sigma_{k}-\sigma_{l}\right)^{2}
\end{array}
$$


In these equations $\Delta=1-\frac{\pi}{6} \xi_{3}, \phi_{1}=\left(1-s \sigma_{k}-e^{-s \sigma_{k}}\right) / s^{2}, \phi_{2}=\left(1-s \sigma_{k}-s^{2} \sigma_{k}^{2} / 2-e^{-s \sigma_{k}}\right) / s^{3}$, and $\xi_{n}=\sum x_{k} \sigma_{k}^{n}$. In approximating equations (14) and (15) we note that the integrands are non-zero over a small range of $s$. In addition we note that the leading terms of the functions $\phi_{n}$ are constants suggesting that we can treat $D$ as being nearly independent of $s$. When D is factored out of the integrals the remaining terms can be integrated analytically.

Preliminary comparisons of the results obtained with this simplification compared to the exact numerical integration results typically agree to within a few percent. Generally, speaking the differences are small indicating that the method is a satisfactory approximation for the size correction. Current work in this area is examining the possibility of incorporating this correction into engineering design equations of state.

Finally we note that work in the third area of corresponding states theory, namely the utilization of integral equation results, is scheduled to start in May.

\section{Summary}

We have presented the results obtained in our investigation of the composition dependence of thermophysical properties. The results obtained during the first eight months of this study are very encouraging and have led to several (submitted) publications. We anticipate that our progress will continue the same or even an accelerated pace during the summer and next academic year. 


\section{Publications and Presentations}

\subsection{Published Manuscripts 8/1/92 - 7/31/93}

1. R. L. Rowley and J. F. Ely. Note on the number dependence of nonequilibrium molecular dynamics simulations of viscosity of structured molecules. J. Chem. Phys., 96:4814-4815, 1992.

2. R. L. Rowley and J. F. Ely. Non-equilibrium molecular dynamics simulations of structured molecules. Part II. Isomeric effects on the viscosity of models for n-hexane, cyclohexane and benzene. Mol. Phys., 75:713-730, 1992.

3. D. G. Friend and J. F. Ely. Thermodynamics of the methanetethane system. Fluid Phase Equilib., 79:77-88, 1992.

4. M. L. Huber and J. F. Ely. Prediction of the viscosity refrigerants of and refrigerant mixtures. Fluid Phase Equilib., 80:239-248, 1992.

5. M. L. Huber, D. G. Friend, and J. F. Ely. Prediction of the thermal conductivity of refrigerants and refrigerant mixtures. Fluid Phase Equilib., 80:249-261, 1992.

\subsection{Manuscripts Submitted or In Press}

1. Computer simulation of the excess Gibbs free energy and chemical potential in Lennard-Jones mixtures with large size ratios., submitted, Mol. Phys., 1993.

2. A modified hard sphere expansion conformal solution theory., submitted, Fluid Phase Equilibria, 1993.

3. A new equation of state for HFC R134a (1,1,1,2-tetrafluoroethane)., submitted, $J$. Chem. Thermodynam., 1993.

4. A predictive extended corresponding states model for pure and mixed refrigerants including a new equation of state for R134a. Int. J. Refrig., Accepted for publication, 1993.

\subsection{Manuscripts in preparation}

1. An analytic equation of state model for mixtures with large size ratios., Ini. J. Thermophys.

2. An extended corresponding states model for carbon dioxide rich mixtures. A.I.Ch.E. Journal. 


\subsection{Presentations, $8 / 1 / 92-7 / 31 / 93$}

1. Thermophysical properties and reactions in supercritical fluids, Chemistry Department, University of Denver, Denver, CO, April 22, 1992.

2. Prediction and correlation of phase equilibria in supercritical solvents, Chemical Engineering Department, Instituto Superior Tecnico, University of Lisbon, Lisbon, Portugal, January 28, 1993.

3. Volume correction factors for custody transfer of natural gas liquids, Gas Processors Association Anrual Convention, San Antonio, TX, March 15, 1993.

4. Development of improved equations of state via molecular dynamics simulations and microscopic theory, Engineering Division, Colorado School of Mines, April 20, 1993.

5. Development of improved equations of state via molecular dynamics simulations and microscopic theory, Chenical Engineering Department, University of Tulsa, Tulsa, OK, April 6, 1993. 


\section{A. SALARIES AND WAGES}

1. James Ely - PI, 1 month AY, 2 mos. summer

2. Graduate Research Assistants (2) - Ph.D., $50 \%$ AY, $100 \%$ summer

subtotal

B. FRINGE BENEFITS

1. $21.5 \%$ of $\mathrm{Al}$

2. 488 of $\mathrm{A} 3$

Subtotal

C. OTHER DIRECT COSTS - I

1. Travel

$\$ 3,000$

2. Publication costs

400

3. Miscellaneous: report preparation, phone, postage, xeroxing

D. MODIFIED TOTAL DIRECT COSTS (MTDC)

E. OTHER DIRECT COSTS - II

1. Permanent equipment

$\$ 7,525$

F. TOTAL DIRECT COSTS

$\$ 83,659$

G. INDIRECT COSTS (52\% of D excluding B2)

32,663

H. TOTAL COST - YEAR 2 


\section{FINANCIAL STATEMENT OF ESTIMATED COSTS}

Statement of Unobligated Balance

Colorado School of Mines

Grant No. DE-FG02-92ER-14294

For the Period August 1, 1992 through July 31, 1993

Total obligated funds from DOE for the current period: $\$ 109,591$

Total anticipated expenditures for the current period: $\$ 109,591$

Anticipated unexpended funds for the current period: $\quad \$ 0$ 

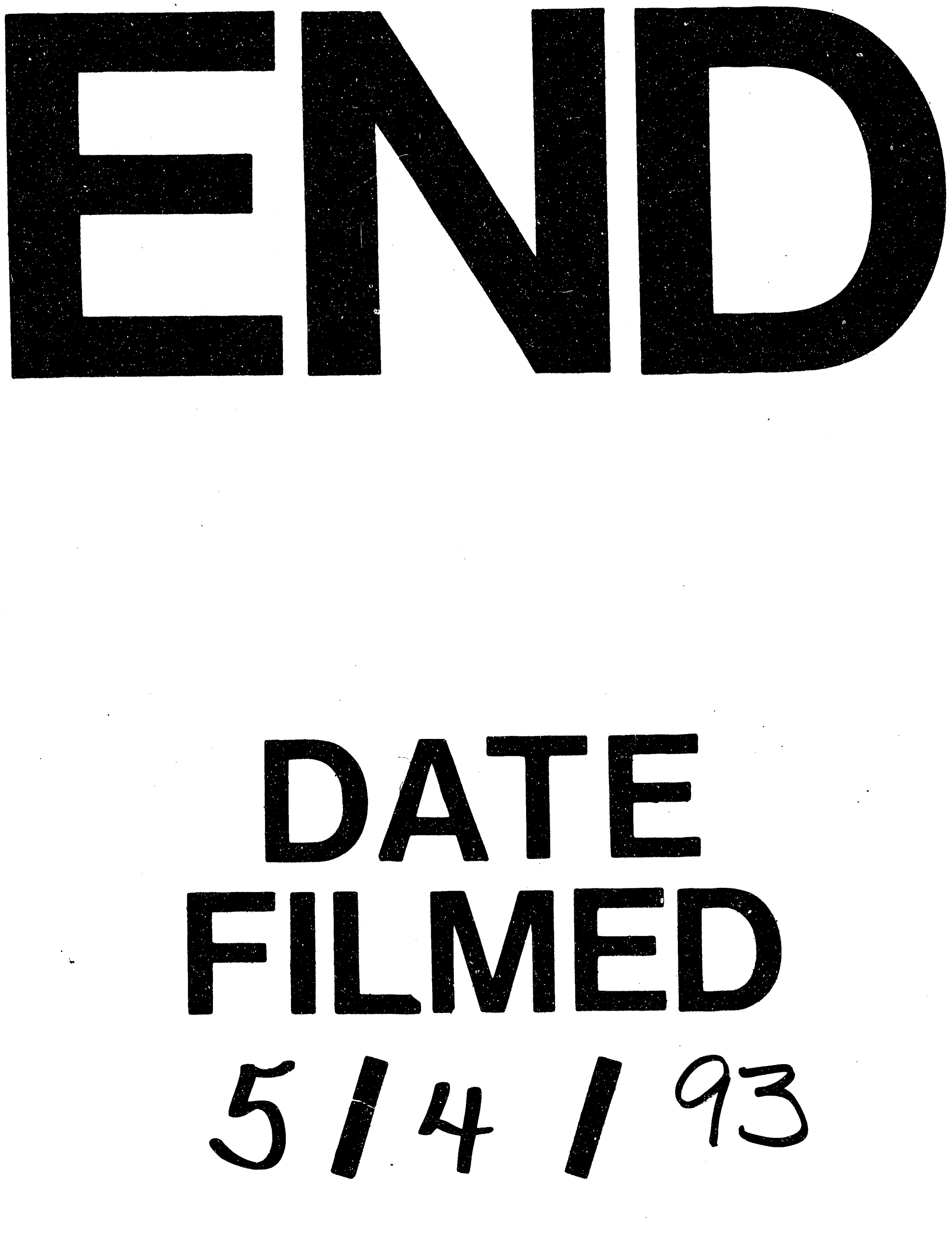

I 
